\title{
Correction to: Lung cancer mortality of residents living near petrochemical industrial complexes: a meta-analysis
}

Cheng-Kuan Lin ${ }^{1}$, Huei-Yang Hung ${ }^{2}$, David C. Christiani ${ }^{1,3}$, Francesco Forastiere ${ }^{4}$ and Ro-Ting Lin ${ }^{5^{*}}$

\section{Correction}

After publication of the article [1], it has been brought to our attention that the original version of this Article contained a typo in the 3rd paragraph of the section 'Review process and data extraction'. It concerns the equation published as " $\operatorname{Var}(\ln R R)=\operatorname{Var}(\ln R 1+\ln R 0)$ ". On the right part, the " + " within the parenthesis should be "-", as defined and derived from the left part. As a result, $\operatorname{Var}(\ln R R)=\operatorname{Var}(\ln R 1+\ln R 0)$ should be revised to $\operatorname{Var}(\ln R R)=\operatorname{Var}(\ln R 1-\ln R 0)$.

\begin{abstract}
Author details
'Department of Environmental Health, Harvard T.H. Chan School of Public Health, 665 Huntington Avenue, Building 1, Room 1401, Boston, MA 02115, USA. ${ }^{2}$ Department of General Medicine, Kaohsiung Medical University Hospital, No. 100, Tzyou 1st Road, Kaohsiung 807, Taiwan. ${ }^{3}$ Department of Epidemiology, Harvard T.H. Chan School of Public Health, 665 Huntington Avenue, Building 1, Room 1401, Boston, MA 02115, USA. ${ }^{4}$ Department of Epidemiology Lazio Regional Health Service, Via Cristoforo Colombo, 112 Rome, Italy. ${ }^{5}$ Department of Occupational Safety and Health, College of Public Health, China Medical University, 91 Hsueh-Shih Road, Taichung 40402, Taiwan.
\end{abstract}

Received: 3 November 2017 Accepted: 8 November 2017

Published online: 15 November 2017

\section{Reference}

1. Lin C, Hung H, Christiani D, Forastiere F, Lin R. Lung cancer mortality of residents living near petrochemical industrial complexes: a meta-analysis. Environ Health. 2017;16:1. doi: 10.1186/s12940-017-0309-2.

\footnotetext{
* Correspondence: roting@mail.cmu.edu.tw

${ }^{5}$ Department of Occupational Safety and Health, College of Public Health,

China Medical University, 91 Hsueh-Shih Road, Taichung 40402, Taiwan
} 